\title{
Factors Associated with the Expression of ACE2 in Human Lung Tissue: Pathological Evidence from Patients with Normal FEV 1 and $\mathrm{FEV}_{\mathrm{I}} / \mathrm{FVC}$
}

\author{
Wuping $\mathrm{Bao}^{1}, *$ \\ Xue Zhang (D) ${ }^{1, *}$ \\ Yubiao Jin $2, *$ \\ Huijuan Hao' \\ Fu Yang ${ }^{3}$ \\ Dongning Yin' \\ Xi Chen ${ }^{3}$ \\ Yishu Xue \\ Lei Han' \\ Min Zhang' \\ 'Department of Respiratory and Critical \\ Care Medicine, Shanghai General \\ Hospital, Shanghai Jiao Tong University \\ School of Medicine, Shanghai, People's \\ Republic of China; ${ }^{2}$ Department of \\ Pathology, Shanghai General Hospital, \\ Shanghai Jiao Tong University School of \\ Medicine, Shanghai, People's Republic of \\ China; ${ }^{3}$ Department of Thoracic Surgery, \\ Shanghai General Hospital, Shanghai Jiao \\ Tong University School of Medicine, \\ Shanghai, People's Republic of China \\ *These authors contributed equally to \\ this work
}

Background: Whether COVID-19 comorbidities and risk factors such as old age, male gender, smoking, obesity, eosinophils and blood types have direct contact with expression of ACE2 and pro-inflammation cytokines in human lung tissues were still unclear.

Patients and Methods: Sixty-four patients with normal $\mathrm{FEV}_{1}$ and $\mathrm{FEV}_{1} / \mathrm{FVC}$ underwent thoracotomy for pulmonary nodules were included. Blinded histological assessments were performed by two pathologists. Clinical features and results of the immunohistochemical staining of ACE2 were collected and analyzed.

Results: ACE2 expressed in alveolar macrophages (most obvious), alveolar epithelia and vascular endothelia, but not in small-airway epithelia. ACE2 expressions are positively related to age $(r=0.26, P=0.040)$, weight $(r=0.43, P<0.001)$, as well as BMI $(r=0.38$, $P=0.002)$, and male patients show higher expressions of ACE2 in lungs $(P<0.05)$. ACE2 expressions are negatively related to peripheral eosinophils $(r=-0.30, P=0.017)$. There was no correlation between ABO blood types and ACE2 expression in normal lung tissues $(P>$ $0.05)$. IL-13 and IL-6R expression in lung tissue increased with age $(r=0.26, P<0.05$, for both).

Conclusion: Our pathological evidences showed that the alveolar epithelia, vascular endothelia, and alveolar macrophages are susceptible in human lungs for SARS-CoV-2 infection. The risk factors such as high body weight/BMI, old age, male gender, and eosinopenia may be related to ACE2 expression in human lungs, and associated with more chance to develop the severe cases. IL-6R expression in lung tissue also increased with age. Therefore, weight control and smoking cessation are essential to reduce the susceptibility of SARS-CoV-2 infection, especially in obesity, old or male patients. Peripheral eosinophils monitor is also quite necessary to detect severe tendency in COVID-19 patients.

Keywords: ACE2, risk factor, BMI, age, sex, eosinophils

\section{Introduction}

A severe acute respiratory syndrome coronavirus 2 (SARS-CoV-2) leading to the coronavirus disease 2019 (COVID-19) was first identified in December 2019 and rapidly swept across the globe. More than 85 million people have been confirmed and more than 1.8 million people dead worldwide (as of December 2020). ${ }^{1}$

It has been estimated that 1 in 5-10 adults with positive COVID-19 requiring hospital admission, ${ }^{2}$ with rates of admission to the intensive care unit (ICU) in China, Europe, and the United States ranging from $5 \%$ to $32 \% .^{3-11}$ The severity of COVID-19 illness poses a great strain to medical resources and a great burden
Correspondence: Min Zhang Department of Respiratory and Critica Care Medicine, Shanghai General Hospital, Shanghai Jiao Tong University School of Medicine, No. 100, Haining Road, Shanghai, 200080, People's Republic of China

Email maggie_zhangmin@I63.com 
to social economy. Therefore, early identification of risk factors of COVID-19 is essential to protect susceptible population, and might help to provide proper prevention, close observation and treatment. Many potential COVID19 comorbidities and risk factors such as coronary artery disease, hypertension, diabetes, COPD, asthma, autoimmune disease, old age, male gender, smoking, and obesity have been demonstrated to affect the process and severity of COVID-19 illness. $^{12,13}$ And the effect of some risk factors, such as eosinophils and blood types in COVID-19 is still controversial.

SARS-CoV-2 attaches and enters host cells first via a transmembrane receptor angiotensin-converting enzyme 2 (ACE2). ACE2 is widely distributed in various human tissues, and in the respiratory system, it is mainly expressed in regions of the sinonasal cavity and the apical surface of alveolar type II cells in pulmonary alveoli. ${ }^{14,15}$ ACE2 protein was found co-localized with TMPRSS2, a co-factor for SARS-CoV-2 entry to cause different degrees of lung injury, including acute respiratory distress syndrome.

ACE2 over-expression is a risk factor for COVID-19 infection and exists in lungs of patients with severe COVID-19. ${ }^{16}$ However, the factors, such as clinical features and commodities, affecting the expression of ACE2 in the respiratory tract and lungs are still unclear. Although reports of pathologic findings in tissue specimens of COVID-19 patients are rapidly emerging, ${ }^{17}$ the ACE2 expression in healthy people is still unclear, which was also important to confirm the established role of ACE2 expression and activity in the initiation and development of COVID-19.

The aims of our present research are to observe the expression of ACE2 and pro-inflammation cytokines in lung tissues from patients with normal forced expiratory volume in 1 second $\left(\mathrm{FEV}_{1}\right)$ and $\mathrm{FEV}_{1}$ /forced vital capacity (FVC), and to explore which risk factors to COVID-19 infection are the potential factors that influence ACE2 expression.

\section{Patients and Methods}

\section{Participants}

This cross-sectional study was approved by the Ethics Committee of Shanghai General Hospital, Shanghai Jiaotong University (No. 2020KY014), and we confirm that the patient data were maintained with confidentiality and complied with the Declaration of Helsinki. We have obtained informed consent from patients to participate in the study before thoracotomy. We enrolled a convenience sample of 64 consecutive patients who met the inclusion criteria, underwent thoracotomy for pulmonary nodules in the Department of Thoracic Surgery from November 2018 to October 2019, and had a complete blood count (CBC), thoracic HRCT, spirometry, ${ }^{18}$ bronchodilation test, fractional exhaled nitric oxide (FENO), ${ }^{19}$ and alveolar component of fractional exhaled nitric oxide (CANO) before partial lobectomy.

Inclusion criteria were as follows: $18-70$ years of age, pulmonary nodules $\leq 3 \mathrm{~cm}$ in diameter, accepting lobectomy, $\mathrm{FEV}_{1} / \mathrm{FVC} \geq 70 \%, \mathrm{FEV}_{1}>80 \%$ predicted, negative bronchodilation test (after inhalation of a $\beta_{2}$ agonist, $\mathrm{FEV}_{1}$ increases $<200 \mathrm{~mL}$ and the $\mathrm{FEV}_{1}$ improvement rate is $<12 \%$ ).

Subjects were excluded if they had had a respiratory tract infection in the past 8 weeks; consistent or variable respiratory symptoms such as cough, expectoration, or dyspnea; used montelukast, long-acting $\beta_{2}$-agonists, theophylline, anticholinergic agents, or inhaled or oral corticosteroids in the previous 4 weeks; had an abnormal CT scan except for nodules; or had concomitant severe systemic diseases. Patients who had more than a 10-pack-year smoking history, who currently smoked, or who had quit less than 2 years before were also excluded.

\section{Data Collected}

Descriptive characteristics and clinical history, including smoking history, were collected.

$\mathrm{FEV}_{1}, \mathrm{FVC}$, maximum expiratory flow at $50 \%$ vital capacity (MEF50), maximal mid-expiratory flow (MMEF), and peak expiratory flow (PEF) were collected and expressed as the percentage of actual value to predicted value. $\mathrm{FEV}_{1} / \mathrm{FVC}$ was calculated as the ratio of two variables. FENO was calculated with a mathematical model by measuring exhaled $\mathrm{NO}$ at a standard flow rate of $50 \mathrm{~mL} / \mathrm{s}$, and CANO was calculated at flow rates of $50 \mathrm{~mL} / \mathrm{s}$ and $200 \mathrm{~mL} / \mathrm{s}$. Measurements of FENO and CANO are given in parts per billion (ppb).

Blood types, the number and percentages of peripheral eosinophils, neutrophils, lymphocytes, and platelets were recorded. The percentage of pneumonectasis of the surgical side quantified with parenchyma analysis at the threshold of -950 HU (GE AW VolumeShare ${ }^{\mathrm{TM}}$ 5, GE Healthcare, Italy) were determined for every patient according to the HRCT (GE Healthcare, Waukesha, WI, USA) images automatically reconstructed in three 
dimensions with volume-rendering technology (Thoracic VCAR software, GE Healthcare, Italy).

Lung tissue resected was at least $5 \mathrm{~cm}$ away from the pulmonary nodules. Blinded histological assessments were performed by two pathologists. The number of macrophages, eosinophils, lymphocytes, and neutrophils per high-power field (HPF) in hematoxylin-eosin (HE)stained slides of lung tissue were counted.

Enzyme-linked immunosorbent assay (ELISA) results of the following cytokines in homogenized lung tissue were also collected: nuclear factor kappaB (NF-kB) (40096, Active Motif, California, USA), total p38 and phosphop38 (ELISA05673-1, Thermo Fisher Scientific, Waltham, USA), interleukin (IL)- $1 \beta$ (EL10028, Anogen, Ontario, Canada), IL-4 (EL10026, Anogen, Ontario, Canada), IL-5 (EL10035, Anogen, Ontario, Canada), IL-6 (EL10023, Anogen, Ontario, Canada), IL-8 (EL10008, Anogen, Ontario, Canada), IL-10 (EL10027, Anogen, Ontario, Canada), IL-13 (EL10054, Anogen, Ontario, Canada), IL-17A (EL10053, Anogen, Ontario, Canada), tumor necrosis factor- $\alpha$ (TNF- $\alpha)$ (EL10019, Anogen, Ontario, Canada), and transforming growth factor- $\beta_{1}\left(\mathrm{TGF}-\beta_{1}\right)(\mathrm{EL} 10029$, Anogen, Ontario, Canada).

\section{Immunohistochemical Staining}

Lung tissue sections were collected for immunohistochemistry. The sections were dewaxed with xylene and rehydrated in methanol. Heat induced epitope retrieval was conducted to reverse the loss of antigenicity. To block endogenous peroxidase activity, slides were submerged in $3 \%$ hydrogen peroxide for 25 minutes at room temperature. Sections were washed with PBS for three times and then incubated with the primary antibody against ACE2 (Thermo Fisher Scientific, MA, USA) for or $1 \mathrm{~h}$ at room temperature. Tissue sections were than incubated with appropriate secondary antibody (Servicebio, Wuhan, China) according to the manufacturer's protocol for 50 minutes at room temperature. Diaminobenzidine was used to visualize the immunoreactivity (DAKO, Denmark), and then the slides were counterstained with Harris' hematoxylin. Finally, the slides were covered by using mounting medium and photographed with a Zeiss microscope (Stuttgart, Germany). Immunoreactivity evaluation was implemented independently by two pathologists who were blinded.

\section{Statistical Analysis}

Analyses were performed with GraphPad Prism version 5.01 (GraphPad Software, San Diego, CA, USA), except for the Spearman correlation coefficient matrix and Spearman rank correlation tests, which were performed with $\mathrm{R}$ version 3.6.1 (Innovative Solutions, St. Louis, MO, USA). The normality of the distribution was checked with the Kolmogorov-Smirnov test. Normally distributed data are expressed as mean \pm standard deviation (SD). Non-normally distributed data are expressed as median and interquartile range (IQR). A Mann-Whitney test was performed to compare inter-group differences between two medians of categorical variables (gender, smoking history, hypertension). A Kruskal-Wallis test was performed to compare inter-group differences between four medians of categorical variables (blood types). The correlation between different variables was determined with Spearman analysis; $r>0.4$ or $<-0.4$ was defined as strong correlation, and $r$ between -0.4 and 0.4 as weak correlation, if $P<0.05$.

The threshold for statistical significance for all analyses was set at $P<0.05$.

\section{Results}

\section{Baseline Characteristics}

Sixty-four patients undergoing thoracotomy for pulmonary nodules were included, with an average age $56.5 \pm 8.96$ years. Thirty-two (50\%) were male and 13 (20.3\%) with smoking history. Most of the pulmonary nodules were adenocarcinomas, except for one squamous cell carcinoma. Twenty-one (32.8\%) had comorbidities: hypertension (12), diabetes (3), rheumatoid arthritis (stable, maintenance medication not required, 1), allergic rhinitis (1), chronic cholecystitis (1), thyroid nodule (postoperative, 1), meningioma (postoperative, 1), renal cyst (1), of breast tumor (postoperative, without chemotherapy, 1), postoperative of hysterectomy (1), kidney stones (1), appendicitis (postoperative, 1), inguinal hernia (1), ovarian cysts (1). Some patients had two or more concurrent comorbidities. All comorbidities were mild and well controlled for at least 6 months.

\section{The Main Location of ACE2 Expression in Lung Tissues}

ACE2 immunostaining was observed in alveolar epithelial cells (Figure 1A), and also vascular endothelial cells (Figure 1B), but not in small airway epithelial cells 

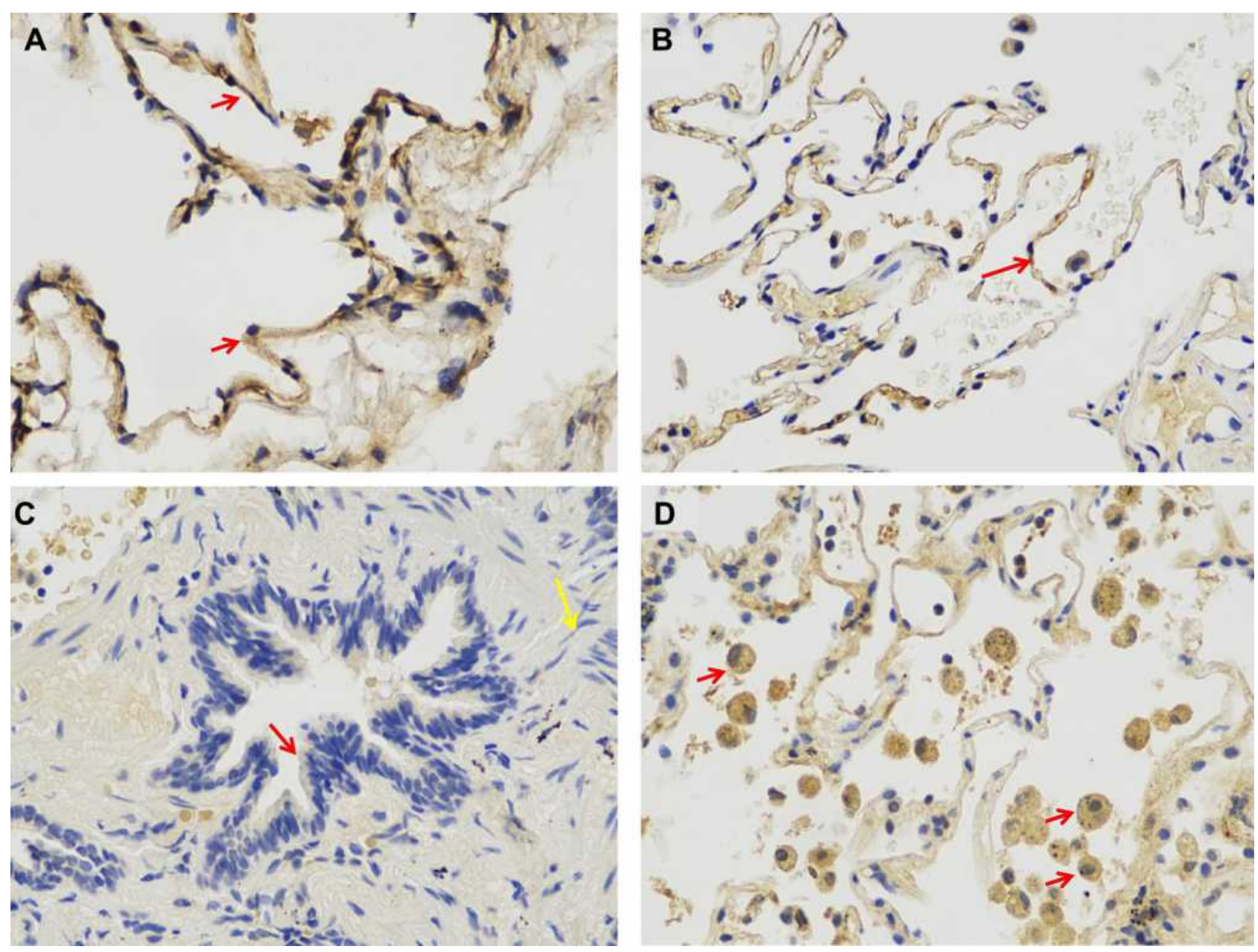

Figure I Representative photomicrographs of immunohistochemistry staining of ACE2 in human lung tissues. (A) alveolar epithelial cells (red arrow); (B) vascular endothelial cells (red arrow); (C) small airway epithelial cells (red arrow) and smooth muscles (yellow arrow); (D) alveolar macrophages (red arrow).

Abbreviation: ACE2, angiotensin-converting enzyme 2.

(Figure 1C). ACE2 expression in alveolar macrophage was also obvious (Figure 1D). Higher ACE2 expression level in alveolar macrophage was proved, compared with alveolar epithelial cell in the same subject (Figure 1D).

\section{Effects of Gender, Smoking History, Hypertension, and Blood Types on the Expression of ACE2 in Lung Tissue}

Smoking history does not influence the ACE2 expressions in lung tissue, with 0.097[0.091-0.109] for patients with smoking history and $0.094[0.087-0.103]$ for non-smoker $(P=0.278$, Figure 2A). ACE2 for male were 0.096[0.091-0.112], higher than female patients $(0.094[0.084-0.100], P=0.044$, Figure 2B). For patients with hypertension, ACE2 expression was 0.110 [0.098-0.121], higher than those with normal blood pressure $(0.093[0.089-0.100], P=0.024$, Figure 2C). There was no significant difference of the ACE2 expression among different ABO blood types ( $P=0.292$, by Kruskal-Wallis test, Figure 2D)

\section{Correlation of ACE2 with Clinical Features}

ACE2 was strong positively correlated with weight $(r=0.43, P<0.001)$ (Figures 3 and 4A). Weak correlations were seen between ACE2 and body mass index (BMI) $(r=0.38, P<0.05)$ (Figures 3 and 4B), height $(r=0.27$, $P<0.05)$ (Figures 3 and $4 \mathrm{C}$ ), age $(r=0.26, P<0.05$ ) (Figures 3 and 4D), macrophages in lung ( $r=0.27$, $P<0.05$ ) (Figures 3 and $4 \mathrm{E}$ ) and peripheral eosinophils $(r=-0.30, P<0.05)$ (Figures 3 and $4 \mathrm{~F}$ ).

ACE2 was not correlated with FENO/CANO, spirometric variables, lymphocytes and neutrophils (peripheral or in lung tissue), eosinophils in lung, platelets, or the percentages of pneumonectasis $(P>0.05$, for all). 

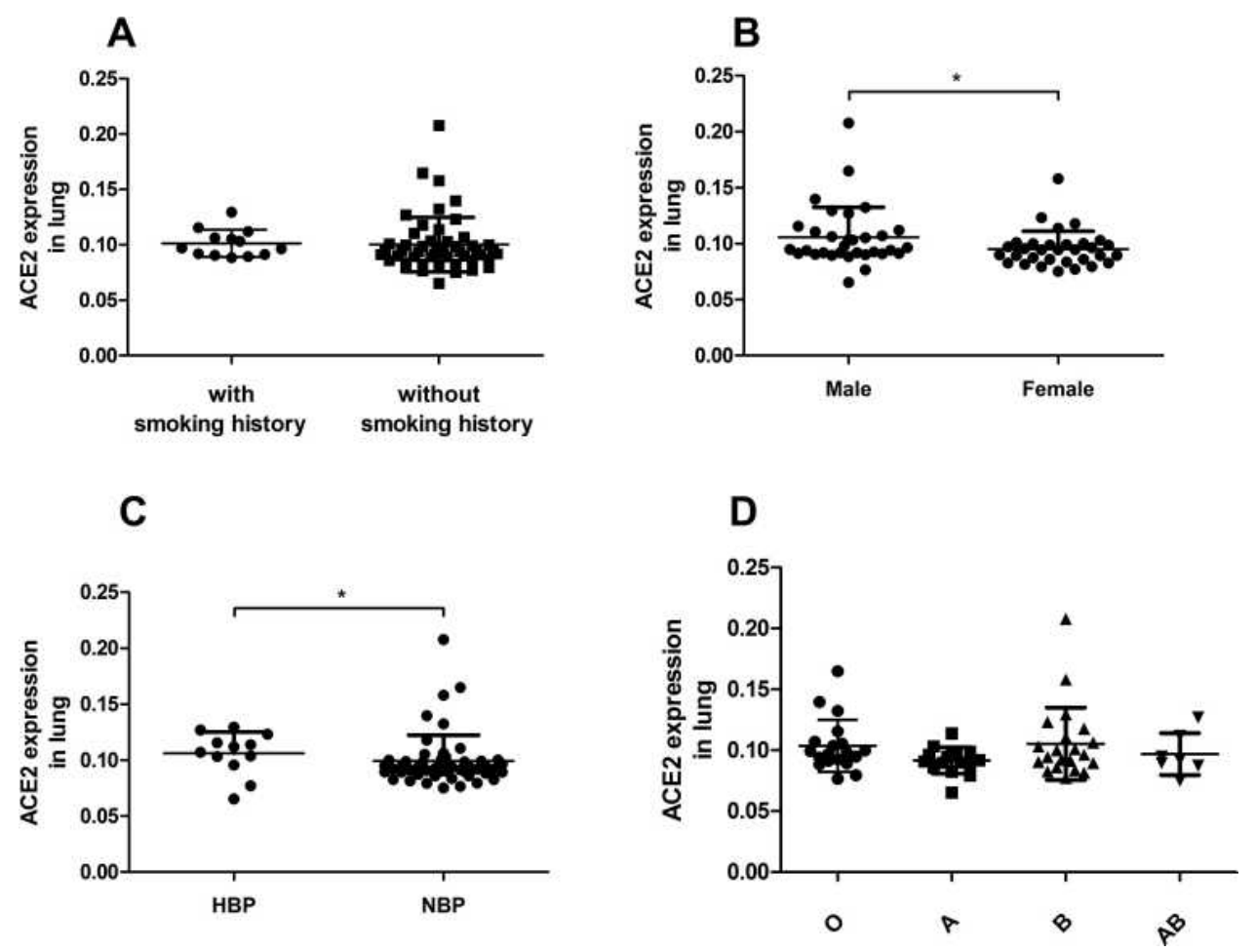

Figure 2 The influence of smoking history (A), gender (B), hypertension (C), and blood types (D) to ACE2 expression in lung tissue. Individual values, mean, and standard deviation are shown. $* P<0.05$.

Abbreviations: ACE2, angiotensin-converting enzyme 2; HBP, high blood pressure; NBP, normal blood pressure.

$P$ values for all correlations are shown in Supplementary Table S1.

\section{Correlation of ACE2 and Age with Pro-Inflammation Cytokines in the Biopsied Tissues}

No statistically significant correlation was found between ACE2 and any of the pro-inflammation cytokines analyzed in lung tissue (IL-6R, TNF- $\alpha$, IL-17A, IL-13, IL-10, IL-8, IL-6, IL-4, IL-1 $\beta$, NF- $\kappa \mathrm{B}: P>0.05$, for all. Figure 5).

Age was found positively correlated with IL-6R and IL13 in lung tissue ( $r=0.26, P<0.05$ for both; Figure 5).

$P$ values for all correlations are shown in Supplementary Table S2.

\section{Discussion}

ACE2 expression in the biopsied lung tissues was reported. In our study, ACE2 expression was found in alveolar epithelial cel, and also vascular endothelial cells, but not in small airway epithelial cells, consistent with severe alveolar injury and non-obvious airway injury proved in COVID-19 patients.
SARS-CoV-2 is proved to infect alveolar macrophages, which in turn respond by producing $\mathrm{T}$ cell chemoattractants, causing alveolitis in which alveolar macrophages containing SARS-CoV-2 and T cells form a positive feedback loop that drives persistent alveolar inflammation. ${ }^{20}$ High expressions of ACE2 in alveolar macrophages also existed in the lungs of mice model, and discovered expressions of ACE2 was dramatically increased on the activated type 1 macrophages (M1), compared with interleukin4-stimulated type 2 macrophages (M2). ${ }^{21}$ Excluding the effect of alveolar macrophage virus-phagocytosis, we also observed the ACE2 expression in alveolar macrophage of human normal lung tissues. Additionally, a positive correlation between ACE2 and macrophage counts in the lungs was found in our research (Supplementary Table S1). Higher ACE2 expression level in alveolar macrophage was proved, compared with alveolar epithelial cell in the same subject. These findings indicated that alveolar macrophages, as the frontline immune cells, may be directly targeted by the SARS-CoV-2 infection and important in the early pathogenesis of COVID-19.

Many COVID-19 risk factors and comorbidities such as old age, male gender, smoking, obesity, coronary artery 


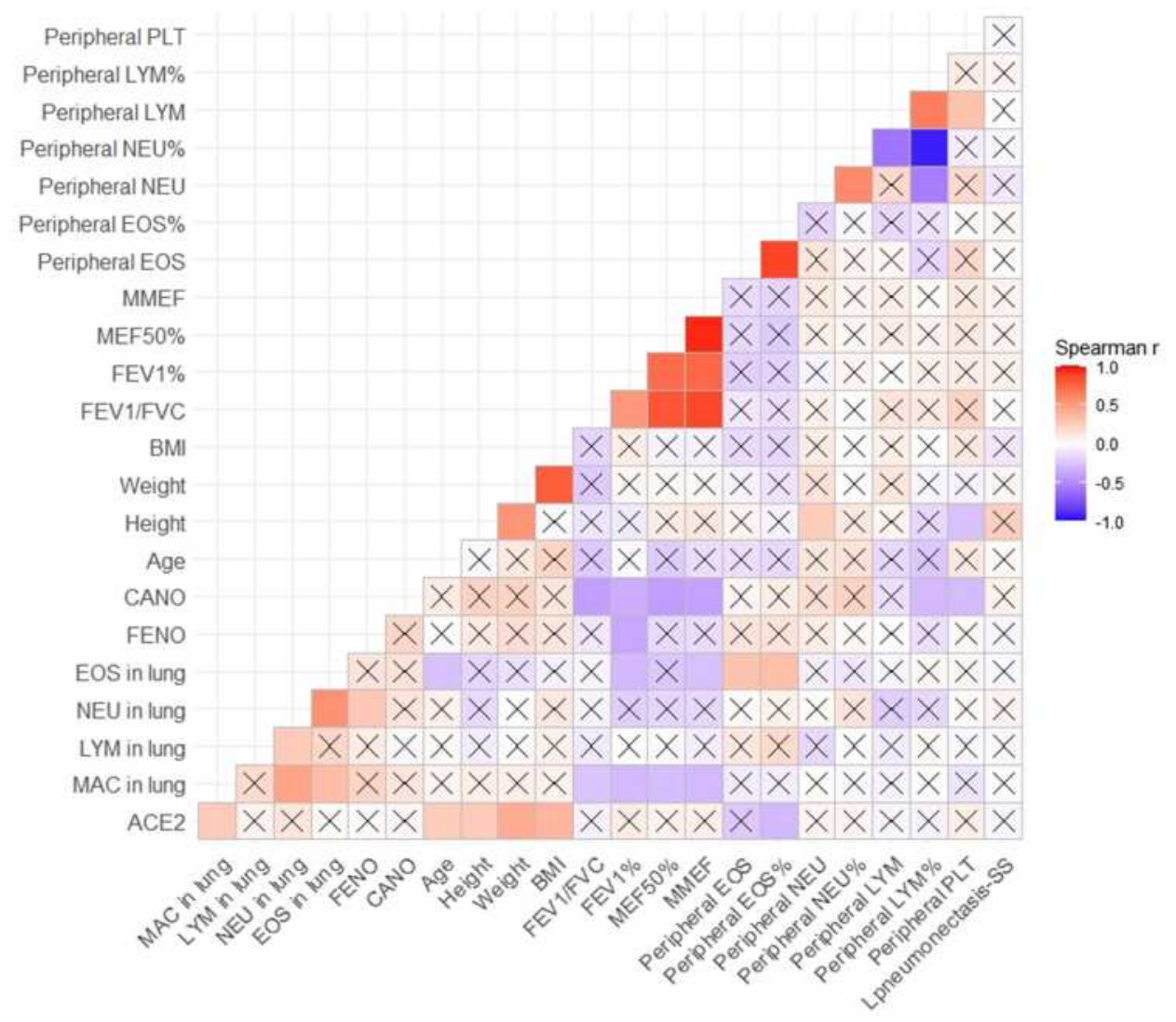

Figure 3 Spearman correlation coefficient matrix and Spearman rank correlation tests for ACE2 and clinical features. $n=64$. ACE2 was positively correlated with weight $(r=0.43$, $P<0.00 \mathrm{I})$, BMI $(r=0.38, P=0.002)$, height $(r=0.27, P=0.034)$, age $(r=0.26, P=0.040)$ and macrophages in lung $(r=0.27, P=0.030)$, and was negatively correlated with peripheral eosinophils $(r=-0.30, P=0.017)$. ACE2 was not correlated with FENO/CANO, spirometric variables, lymphocytes and neutrophils (peripheral or in lung tissue), eosinophils in lung, platelets, or the percentages of pneumonectasis $(P>0.05$, for all). A cross indicates no statistical significance. $P$ values are shown in Supplementary Table $S I$.

Abbreviations: PLT, platelets; LYM, lymphocytes; NEU, neutrophils; EOS, eosinophil; MMEF, percentage of actual value to predicted value of maximum mid-expiratory flow; MEF50, percentage of actual value to predicted value of forced expiratory flow at $50 \%$ of forced vital capacity; $\mathrm{FEV}_{1}$, forced expiratory volume in I second; FVC, forced vitalcapacity; BMI, body mass index; CANO, alveolar concentration of nitric oxide; FENO, fractional exhaled nitric oxide; MAC, macrophages; ACE2, angiotensin-converting enzyme 2; SS, surgical side.

disease, hypertension, diabetes, COPD, severe asthma, and autoimmune disease have been reported that effect the progress and severity of COVID-19. ${ }^{13}$ Obesity, an essential risk factor of COVID-19, contributes to a high morbidity, hospitality, risk of intensive care, mechanical ventilation, venous thromboembolism, dialysis, and mortality. ${ }^{22-25}$ Radzikowska et $\mathrm{al}^{26}$ studied gene expression and co-expression of ACE2, CD147, CD26 in primary human bronchial epithelial cells, bronchial and skin biopsies, bronchoalveolar lavage fluid, whole blood, and immune cells. Obesity led to the higher expression of ACE2-related genes in the bronchial biopsy and BAL. ${ }^{26}$ Increased ACE2 bronchial epithelial expression in COPD patients with overweight was also proved, compared to those normal weight patients. ${ }^{27}$ But the expression of ACE2 in the respiratory bronchiole and the alveoli, which covered majority of the breathing area, was still unclear. A mice model research demonstrated that diet- induced obesity mice exhibit markedly increased ACE2 gene expression in the lung tissue. ${ }^{28}$ ACE2 was reported with high expression in white adipose tissues, which may act as a reservoir of SARS-CoV-2 with increased shedding, immune activation, and pro-inflammatory cytokine amplification. Weight loss induced decline of subcutaneous adipose ACE2 expression. ${ }^{29}$ Whether subcutaneous adipose ACE2 Expression was directly involved in the occurrence and development of COVID-19 infection remains to be studied. We demonstrated the positive correlations between ACE2 respiratory bronchiole and alveoli expression and weight, as well as BMI. Our results provide more pathological evidences of overweight/obesity as a great risk of COVID-19 infection.

A number of studies have revealed that old age is associated with higher COVID-19 morbidity and disease severity and also a very strong risk factor of COVID-19 deaths. ${ }^{30}$ We found that ACE2 expressions are positively 
A

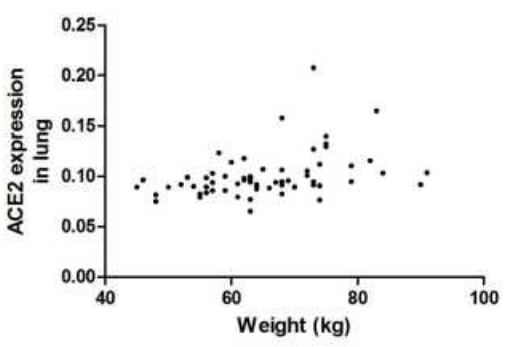

D

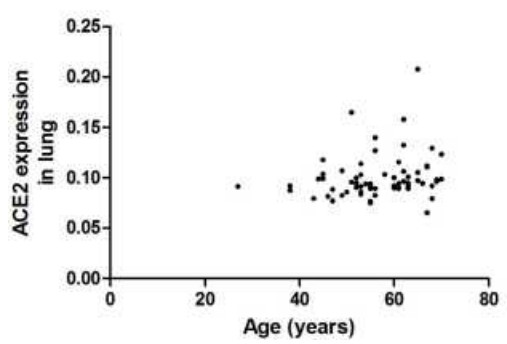

B

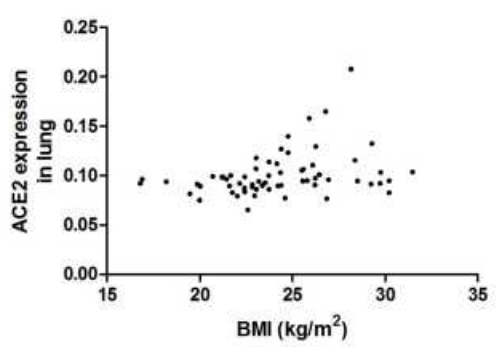

E

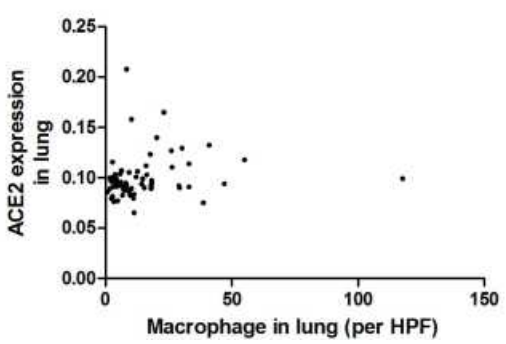

C

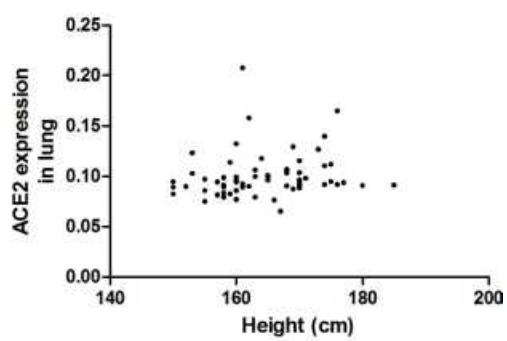

F

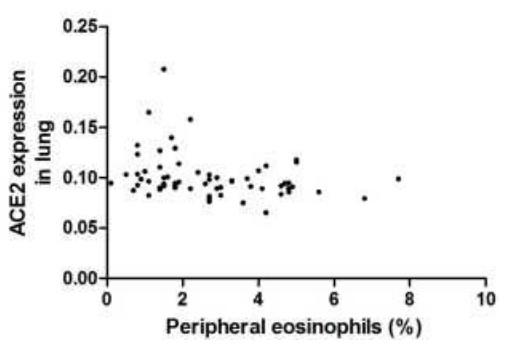

Figure 4 Correlation between ACE2 expressions and (A) weight, (B) BMI, (C) height, (D) age, (E) macrophages in lung and (F) peripheral eosinophils $(\%) . n=64 ;(\mathbf{A})$ $r=0.43(95 \% \mathrm{Cl}, 0.20$ to 0.61 ), $P<0.00 \mathrm{I}$; (B) $r=0.38$ (95\% Cl, 0.14 to 0.58 ), $P=0.002 ;$ (C) $r=0.27$ (95\% Cl, 0.01 to 0.49 ), $P=0.034 ;$ (D) $r=0.26$ (95\% Cl, 0.01 to 0.48 ), $P=0.040 ;(E) r=0.27(95 \% \mathrm{Cl}, 0.02$ to 0.49$), P=0.030 ;(\mathbf{F}) r=-0.299$ ( $95 \% \mathrm{Cl},-0.51$ to -0.05$), P=0.017$.

Abbreviations: ACE2, angiotensin-converting enzyme 2; BMI, body mass index; HPF, high-power field.

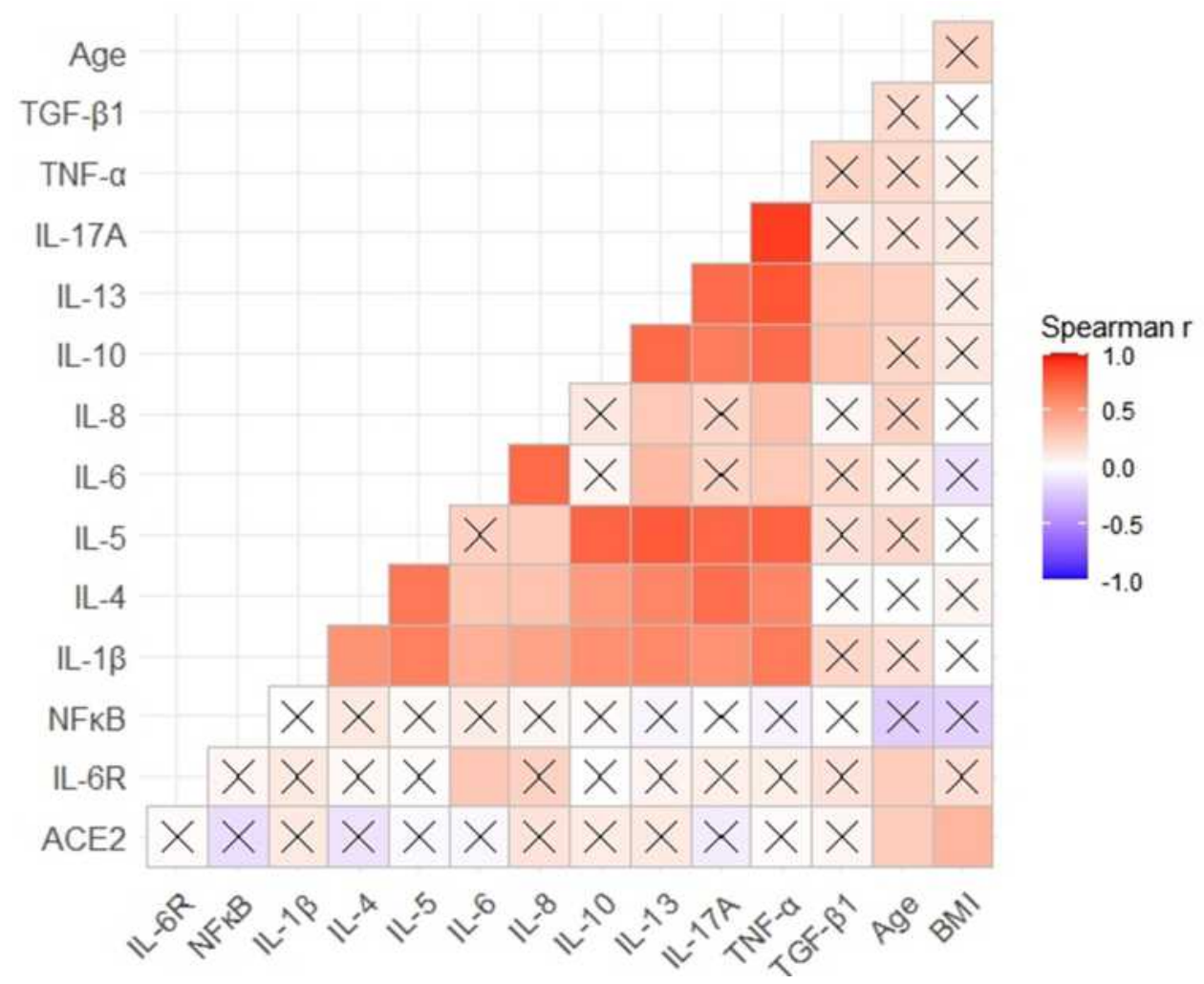

Figure 5 Correlation of ACE2 and age with pro-inflammation cytokines in the biopsied tissues. $n=64$. No statistically significant correlation was found between ACE2 and any of the pro-inflammation cytokines analyzed in lung tissue (IL-6R, TNF- $\alpha$, IL-I7A, IL-13, IL-I0, IL-8, IL-6, IL-4, IL-I $\beta$, NF- $\kappa B$ : P>0.05, for all). Age was found positively correlated with IL-6R and IL-13 in lung tissue. ( $r=0.26, P<0.05$ for both).

Abbreviations: ACE2, angiotensin-converting enzyme 2; IL-6R, interleukin-6 receptor; TNF- $\alpha$, tumor necrosis factor- $\alpha$, IL-I7A, interleukin- I7A; IL-I3, interleukin-I3; ILI0, interleukin- I0; IL-8, interleukin-8; IL-6, interleukin-6; IL-4, interleukin-4; IL-I $\beta$, interleukin-I $\beta$; NF- $\kappa B$, nuclear factor- $\kappa B$. 
related to age. IL-13, a type 2 (T2) cytokine, was recently observed to suppress ACE2 expression and increase transmembrane protease serine 2 (TMPRSS2) expression of airway epithelial cells in T2 asthma and atopy patients, making it difficult to predict how T2 inflammation might affect risk for COVID-19. ${ }^{31}$ In addition, IL-13 also induced airway inflammation, airway injury, obstruction, pulmonary fibrosis, and exacerbate acute lung injury. ${ }^{32,33}$ IL-6 is a key cytokine that mediates immune disorder induced by SARS-CoV-2 and may play an important role in the immune mechanism of patients with severe COVID19 via ligation to IL-6R ${ }^{34,35}$ IL-13 and IL-6R expression in lung tissue also increased with age in our study, indicating that old age may increase pro-inflammation cytokine IL-13 expression and may partially explain that old age population was more susceptible to SARS-CoV-2 infection. On the other side, as the two-side effect of IL-13, the exact effect of IL-13 on SARS-CoV-2 infection is uncertain and needs further research.

Male gender is a risk factor for more severe COVID19, including death. Male patients are almost twice as likely as female to die from COVID-19. ${ }^{36,37}$ We also found that male patients show higher expressions of ACE2 in lungs, which might explain the differences of COVID-19 morbidity and disease severity among different sex. There was no difference of ACE2 expression between non-smokers and ex-smokers in our study $(P>0.05)$, largely because patients with non-smoking and who had less than a 10-pack-year smoking history and had quit more than 2 years were enrolled in our study, indicating smoking cessation at least for 2 years may potentially help to reduce the risk of COVID-19 infection.

Although the effect of eosinophils in COVID-19 is controversial, evidence is emerging that eosinopenia may serve as an effective prognostic indicator for more severe COVID-19. An analysis for 72,314 COVID-19 cases in China reported that respiratory allergy and asthma have not been identified as significant risk factors for severe COVID-19 illness. $^{38}$ Our previous study interestingly observed that $71.7 \%$ COVID-19 inpatients had decreased, even disappeared circulating eosinophil counts at the onset of disease. Eosinopenia at admission indicated more severe infection and longer hospitalization. ${ }^{39}$ In this study, ACE2 expressions in the lungs were negatively related to peripheral eosinophils, providing pathological evidence for the high susceptibility of patients with eosinopenia to SARS-CoV-2. As a result, this finding indicate that peripheral eosinophils may act as a protect role in
SARS-CoV-2 infection. In this study, ACE2 expressions in the lungs were negatively related to peripheral eosinophils, providing pathological evidence for the high susceptibility of patients with eosinopenia and a protective role of eosinophils in SARS-CoV-2 infection.

The relationship between blood type and COVID-19 is still unclear. Peng GW and colleagues were the first to find that type A blood was associated with a higher risk for COVID-19, whereas type O blood was associated with a lower risk. ${ }^{40}$ Then scientists from Europe and Australia conducted a genome-wide association study and found that association signal at locus $9 \mathrm{q} 34.2$ carries the gene that determines the $\mathrm{ABO}$ blood type. Consistent with previous findings from China, they also found that type A had a higher risk of developing severe COVID-19, while those with type $\mathrm{O}$ had a lower risk. ${ }^{41}$ Recently, a population-based cohort study including 225556 persons reported the $\mathrm{O}$ and $\mathrm{Rh}$ blood groups may be associated with a slightly lower risk for SARS-CoV-2 infection and severe COVID-19 illness. ${ }^{42}$ However, the research found that the risk of intubation was decreased for type $\mathrm{A}$ and increased for types $\mathrm{AB}$ and $\mathrm{B}$, compared with type $O$, while risk of death was decreased among $\mathrm{A}$ and $\mathrm{B}$ types and increased in $\mathrm{AB}$ type, although no correlation was found between blood type and risk of intubation or death in COVID-19 patients. ${ }^{43,44}$ In our study, we observed whether ACE2 expression in normal lung tissue is correlated with the $\mathrm{ABO}$ blood type. We found no correlation between ACE2 expression and ABO blood type, despite the small sample size. Whether blood type is linked to COVID-19 risk and whether ACE2 contributes to those correlation are still extremely lack of evidence, and further investigation is needed to be conducted.

Besides, we found ACE2 was not correlated with FENO/CANO, spirometric variables, lymphocytes and neutrophils (peripheral or in lung tissue), eosinophils in lung, platelets, or the percentages of pneumonectasis ( $P>0.05$ for all, Figure 3; $P$ values for all correlations are shown in Supplementary Table S1). And no statistically significant correlation was found between ACE2 and any of the pro-inflammation cytokines analyzed in lung tissue (IL-6R, TNF- $\alpha$, IL-17A, IL-10, IL-8, IL-4, IL-1 $\beta$, NF-кB: $P>0.05$, for all. Figure 5, $P$ values for all correlations are shown in Supplementary Table S2).

To overcome some limitations of our current study, larger-scale and further mechanisms research should be performed to validate our inspection results. 


\section{Conclusions}

Our pathological evidences showed that the alveolar epithelia, vascular endothelia, and alveolar macrophages are susceptible cells in human lungs for SARS-CoV-2 infection. The risk factors such as high body weight/BMI, old age, male gender, and eosinopenia may be related to ACE2 expression in human lungs, and associated with more chance to develop the severe cases. IL-6R expression in lung tissue also increased with age. Therefore, weight control and smoking cessation are essential to reduce the susceptibility of SARS-CoV-2 infection, especially in those population with obesity, old age, and male gender. Peripheral eosinophils monitor is also quite necessary to detect severe tendency in COVID-19 patients.

\section{Abbreviations}

ACE2, angiotensin-converting enzyme 2; BMI, body mass index; CBC, complete blood count; CANO, alveolar component of fractional exhaled nitric oxide; COVID-19, coronavirus disease 2019; ELISAs, enzyme-linked immunosorbent assays; $\mathrm{FEV}_{1}$, forced expiratory volume in 1 second; $\mathrm{FVC}, \mathrm{FEV}_{1}$ /forced vital capacity; FENO, fractional exhaled nitric oxide; HE, hematoxylin-eosin; HPF, high-power field; ICU, Intensive care unit; IL, interleukin; IQR, interquartile range; MEF50, maximum expiratory flow at $50 \%$ vital capacity; MMEF, maximal mid-expiratory flow; NF- $\kappa \mathrm{B}$, nuclear factor kappa-B; PEF, peak expiratory flow; ppb, parts per billion; SARS-CoV-2, severe acute respiratory syndrome coronavirus 2 ; SD, sandard deviation; TGF- $\beta_{1}$, transforming growth factor- $\beta_{1}$; TMPRSS2, transmembrane protease serine 2 ; TNF- $\alpha$, tumor necrosis factor- $\alpha$.

\section{Data Sharing Statement}

The data sets used and/or analyzed during the current study are available from the corresponding author on reasonable request.

\section{Ethics Approval}

This cross-sectional study was approved by the Ethics Committee of Shanghai General Hospital, Shanghai Jiaotong University (No. 2020KY014).

\section{Author Contributions}

All authors made substantial contributions to conception and design, acquisition of data, or analysis and interpretation of data; took part in drafting the article or revising it critically for important intellectual content; agreed to submit to the current journal; gave final approval of the version to be published; and agree to be accountable for all aspects of the work.

\section{Funding}

The study was supported by National Natural Science Foundation of China (Grant No. 81873402, and 81800020); Appropriate Technology Application Program of Shanghai Municipal Health System (Grant No.2019SY042); Scientific and Technological Innovation program funded by the Science and Technology Commission of Shanghai municipality (Grant No. 20Y11902400); Program of Shanghai Municipal Health System (Grant No.201740039); Shanghai Jiao Tong University scientific research fund for COVID-19 prevention and control (Grant NO. YG2020YQ22) and Zhejiang University special scientific research fund for COVID-19 prevention and control (Grant NO. 2020XGZX009). All the grants were awarded to Min Zhang, except No. 81800020 , which was awarded to Wuping Bao.

\section{Disclosure}

All authors reported no conflicts of interest for this work and no potential conflicts of interest with any companies or organizations whose products or services may be discussed in this article.

\section{References}

1. World Health Organization.Coronavirus disease (COVID-19) pandemic. Available from: https:/www.who.int/emergencies/diseases/ novel-coronavirus-2019. Accessed April 9, 2021.

2. Verity R, Okell LC, Dorigatti I, et al. Estimates of the severity of coronavirus disease 2019: a model-based analysis. Lancet Infect Dis. 2020;20(6):669-677. doi:10.1016/S1473-3099(20)30243-7

3. Huang C, Wang Y, Li X, et al. Clinical features of patients infected with 2019 novel coronavirus in Wuhan, China. Lancet. 2020;395 (10223):497-506. doi:10.1016/S0140-6736(20)30183-5

4. Guan WJ, Ni ZY, Hu Y, et al. China Medical Treatment Expert Group for Covid-19. Clinical characteristics of coronavirus disease 2019 in China. $N$ Engl J Med. 2020;382(18):1708-1720. doi:10.1056/ NEJMoa2002032

5. Chen N, Zhou M, Dong X, et al. Epidemiological and clinical characteristics of 99 cases of 2019 novel coronavirus pneumonia in Wuhan, China: a descriptive study. Lancet. 2020;395 (10223):507-513. doi:10.1016/S0140-6736(20)30211-7

6. Onder G, Rezza G, Brusaferro S. Case-fatality rate and characteristics of patients dying in relation to COVID-19 in Italy. JAMA. 2020;323 (18):1775-1776. doi:10.1001/jama.2020.4683

7. Richardson S, Hirsch JS, Narasimhan M, et al.; and the Northwell COVID-19 Research Consortium. Presenting characteristics, comorbidities, and outcomes among 5700 patients hospitalized with COVID-19 in the New York City Area. JAMA. 2020;323 (20):2052-2059. doi:10.1001/jama.2020.6775

8. No authors listed. Clarification of mortality rate and data in abstract, results, and table 2. JAMA. 2020;323(20):2098. doi:10.1001/ jama.2020.7681 
9. Remuzzi A, Remuzzi G. COVID-19 and Italy: what next? Lancet. 2020;395(10231):1225-1228. doi:10.1016/S0140-6736(20)30627-9

10. Grasselli G, Zangrillo A, Zanella A, et al.; COVID-19 Lombardy ICU Network. Baseline characteristics and outcomes of 1591 patients infected with SARS-CoV-2 admitted to ICUs of the Lombardy Region, Italy. JAMA. 2020;323(16):1574-1581. doi:10.1001/jama.2020.5394

11. Docherty AB, Harrison EM, Green CA, et al.; ISARIC4C investigators. Features of 20133 UK patients in hospital with covid-19 using the ISARIC WHO Clinical Characterisation Protocol: prospective observational cohort study. BMJ. 2020;369:m1985. doi:10.1136/bmj.m1985

12. Williamson EJ, Walker AJ, Bhaskaran K, et al. Factors associated with COVID-19-related death using OpenSAFELY. Nature. 2020;584 (7821):430-436. doi:10.1038/s41586-020-2521-4

13. Goyal P, Choi JJ, Pinheiro LC, et al. Clinical characteristics of Covid-19 in New York City. $N$ Engl $J$ Med. 2020;382 (24):2372-2374. doi:10.1056/NEJMc2010419

14. Hoffmann M, Kleine-Weber H, Schroeder S, et al. SARS-CoV-2 cell entry depends on ACE2 and TMPRSS2 and is blocked by a clinically proven protease inhibitor. Cell. 2020;181(2):271-280. e8. doi:10.1016/j.cell.2020.02.052

15. Bezara O, Thurman A, Pezzulo AA, et al. Heterogeneous expression 1 of the SARS-Coronavirus-2 receptor ACE2 in the human respiratory tract. EBioMedicine. 2020;60:102976. doi:10.1016/j.ebiom.2020.102976

16. Chua RL, Lukassen S, Trump S, et al. COVID-19 severity correlates with airway epithelium-immune cell interactions identified by singlecell analysis. Nat Biotechnol. 2020;38(8):970-979. doi:10.1038/ s41587-020-0602-4

17. Nie X, Qian LJ, SUn R, et al. Multi-organ proteomic landscape of COVID-19 autopsie. Cell. 2021;184(3):775-791.e14. doi:10.1016/j. cell.2021.01.004

18. Miller A, Enright PL. PFT interpretive strategies: American Thoracic Society/European Respiratory Society 2005 guideline gaps. Respir Care. 2012;57(1):127-133; discussion 133-135. doi:10.4187/ respcare. 01503

19. Dweik RA, Boggs PB, Erzurum SC, et al. An official ATS clinical practice guideline: interpretation of exhaled nitric oxide levels (FENO) for clinical applications. Am J Respir Crit Care Med. 2011;184(5):602-615. doi:10.1164/rccm.9120-11ST

20. Grant RA, Morales-Nebreda L, Markov NS, et al.; NU SCRIPT Study Investigators. Circuits between infected macrophages and $\mathrm{T}$ cells in SARS-CoV-2 pneumonia. Nature. 2021;590 (7847):635-641. doi:10.1038/s41586-020-03148-w

21. Song $\mathrm{X}, \mathrm{Hu} \mathrm{W}, \mathrm{Yu} \mathrm{H}$, et al. Little to no expression of angiotensin-converting enzyme-2 on most human peripheral blood immune cells but highly expressed on tissue macrophages. Cytometry A. 2020. doi:10.1002/cyto.a. 24285

22. Kass DA. COVID-19 and severe obesity: a big problem? Ann Intern Med. 2020;173(10):840-841. doi:10.7326/M20-5677

23. Tartof SY, Qian L, Hong V, et al. Obesity and mortality among patients diagnosed with COVID-19: results from an integrated health care organization. Ann Intern Med. 2020;173(10):773-781. doi:10.7326/M20-3742

24. Anderson MR, Geleris J, Anderson DR, et al. Body mass index and risk for intubation or death in SARS-CoV-2 Infection: a Retrospective Cohort Study. Ann Intern Med. 2020;173 (10):782-790. doi:10.7326/M20-3214

25. Hendren NS, Lemos JA, Ayers C, et al. Association of body mass index and age with morbidity and mortality in patients hospitalized with COVID-19: results From the American Heart Association COVID-19 Cardiovascular Disease Registry. Circulation. 2021;143 (2):135-144. doi:10.1161/CIRCULATIONAHA.120.051936

26. Radzikowska U, Ding M, Tan G, et al. Distribution of ACE2, CD147, CD26, and other SARS-CoV-2 associated molecules in tissues and immune cells in health and in asthma, COPD, obesity, hypertension, and COVID-19 risk factors. Allergy. 2020;75(11):2829-2845. doi:10.1111/all.14429
27. Higham A, Singh D. Increased ACE2 expression in bronchial epithelium of COPD patients who are overweight. Obesity. 2020;28 (9):1586-1589. doi:10.1002/oby.22907

28. Al Heialy S, Hachim MY, Senok A, et al. Regulation of angiotensin converting enzyme 2 (ACE2) in obesity: implications for COVID-19. Front Physiol. 2020;11:555039. doi:10.3389/fphys.2020.555039

29. Li L, Spranger L, Soll D, et al. Metabolic impact of weight loss induced reduction of adipose ACE-2 - Potential implication in COVID-19 infections? Metabolism. 2020;113:154401. doi:10.1016/ j.metabol.2020.154401

30. Mallapaty S. The coronavirus is most deadly if you are older and male - new data reveal the risks. Nature. 2020;585(7823):16-17. doi:10.1038/d41586-020-02483-2

31. Kimura H, Francisco D, Conway M, et al. Type 2 inflammation modulates ACE2 and TMPRSS2 in airway epithelial cells. $J$ Allergy Clin Immunol. 2020;146(1):80-88.e8. doi:10.1016/j. jaci.2020.05.004

32. Kang MJ, Choi JM, Kim BH, et al. IL-18 induces emphysema and airway and vascular remodeling via IFN- $\gamma$, IL-17A, and IL-13. Am J Respir Crit Care Med. 2012;185(11):1205-1217. doi:10.1164/ rccm.201108-1545OC

33. Laoukili J, Perret E, Willems T, et al. IL-13 alters mucociliary differentiation and ciliary beating of human respiratory epithelial cells. J Clin Invest. 2001;108(12):1817-1824. doi:10.1172/JCI200113557

34. Moore JB, June CH. Cytokine release syndrome in severe COVID-19. Science. 2020;368(6490):473-474. doi:10.1126/science. abb8925

35. Giamarellos-Bourboulis EJ, Netea MG, Rovina N, et al. Complex immune dysregulation in COVID-19 patients with severe respiratory failure. Cell Host Microbe. 2020;27(6):992-1000.e3. doi:10.1016/j. chom.2020.04.009

36. Park MD. Sex differences in immune responses in COVID-19. Nat Rev Immunol. 2020;20(8):461. doi:10.1038/s41577-020-0378-2

37. Takahashi T, Ellingson MK, Wong $P$, et al. Sex differences in immune responses that underlie COVID-19 disease outcomes. Nature. 2020;588(7837):315-320. doi:10.1038/s41586-020-2700-3

38. Wu Z, McGoogan JM. Characteristics of and important lessons from the Coronavirus Disease 2019 (COVID-19) Outbreak in China: summary of a report of 72314 cases from the Chinese center for disease control and prevention. JAMA. 2020;323(13):1239-1242. doi:10.1001/jama.2020.2648

39. Xie G, Ding F, Han L, et al. The role of peripheral blood eosinophil counts in COVID-19 patients. Allergy. 2021;76(2):471-482. doi:10.1111/all.14465

40. Zhao J, Yang Y, Huang H, et al. Relationship between the ABO Blood Group and the COVID-19 Susceptibility. Clin Infect Dis. 2020; ciaa1150.

41. Ellinghaus D, Degenhardt F, Bujanda L, et al.; Severe Covid-19 GWAS Group. Genomewide Association Study of Severe Covid-19 with Respiratory Failure. N Engl J Med. 2020;383(16):1522-1534.

42. Ray JG, Schull MJ, Vermeulen MJ, Park AL. Association between $\mathrm{ABO}$ and $\mathrm{Rh}$ blood groups and SARS-CoV-2 infection or severe COVID-19 illness: a Population-Based Cohort Study. Ann Intern Med. 2020;M20-4511.

43. Latz CA, DeCarlo C, Boitano L, et al. Blood type and outcomes in patients with COVID-19. Ann Hematol. 2020;99(9):2113-2118. doi:10.1007/s00277-020-04169-1

44. Zietz M, Zucker J, Tatonetti NP. Associations between blood type and COVID-19 infection, intubation, and death. Nat Commun. 2020;11(1):5761. doi:10.1038/s41467-020-19623-x 


\section{Publish your work in this journal}

The Journal of Inflammation Research is an international, peerreviewed open-access journal that welcomes laboratory and clinical findings on the molecular basis, cell biology and pharmacology of inflammation including original research, reviews, symposium reports, hypothesis formation and commentaries on: acute/chronic inflammation; mediators of inflammation; cellular processes; molecular

mechanisms; pharmacology and novel anti-inflammatory drugs; clinical conditions involving inflammation. The manuscript management system is completely online and includes a very quick and fair peerreview system. Visit http://www.dovepress.com/testimonials.php to read real quotes from published authors.

Submit your manuscript here: https://www.dovepress.com/journal-of-inflammation-research-journal 